\author{
OAK RIDGE \\ NATIONAL LABORATORY
}

MANAGED BY UT-BATTELLE

FOR THE DEPARTMENT OF ENERGY
ORNL/TM-2008/135

\section{Data Compilation for AGR-2 UCO Variant Coated Particle Batch G73J-14-93074A}

John D. Hunn

October 2008

This report was prepared as an account of work sponsored by an agency of the United States Government. Neither the United States Government nor any agency thereof, or any of their employees, makes any warranty, expressed or implied, or assumes any legal liability or responsibility for any third party's use, or the results of such use, of any information, apparatus, product or process disclosed in this report, or represents that its use by such third party would not infringe privately owned rights. 


\section{DOCUMENT AVAILABILITY}

Reports produced after January 1, 1996, are generally available free via the U.S. Department of Energy (DOE) Information Bridge.

Web site http://www.osti.gov/bridge

Reports produced before January 1, 1996, may be purchased by members of the public from the following source.

National Technical Information Service

5285 Port Royal Road

Springfield, VA 22161

Telephone 703-605-6000 (1-800-553-6847)

TDD 703-487-4639

Fax 703-605-6900

E-mail info@ntis.fedworld.gov

Web site http://www.ntis.gov/support/ordernowabout.htm

Reports are available to DOE employees, DOE contractors, Energy Technology Data Exchange (ETDE) representatives, and International Nuclear Information System (INIS)

representatives from the following source.

Office of Scientific and Technical Information

P.O. Box 62

Oak Ridge, TN 37831

Telephone 865-576-8401

Fax 865-576-5728

E-mail reports@adonis.osti.gov

Web site http://www.osti.gov/contact.html

This report was prepared as an account of work sponsored by an agency of the United States Government. Neither the United States Government nor any agency thereof, nor any of their employees, makes any warranty, express or implied, or assumes any legal liability or responsibility for the accuracy, completeness, or usefulness of any information, apparatus, product, or process disclosed, or represents that its use would not infringe privately owned rights. Reference herein to any specific commercial product, process, or service by trade name, trademark, manufacturer, or otherwise, does not necessarily constitute or imply its endorsement, recommendation, or favoring by the United States Government or any agency thereof. The views and opinions of authors expressed herein do not necessarily state or reflect those of the United States Government or any agency thereof. 


\title{
Data Compilation for AGR-2 UCO Variant Coated Particle Batch G73J-14-93074A
}

\author{
John D. Hunn \\ Oak Ridge National Laboratory
}

Coated particle fuel batch G73J-14-93074A was produced by the Babcock and Wilcox Company $(\mathrm{B} \& \mathrm{~W})$ for possible selection as UCO variant fuel for the Advanced Gas Reactor Fuel Development and Qualification Program's AGR-2 irradiation test. Batch G73J-14-93074A is a single batch of TRISO-coated $425 \mu \mathrm{m}$ nominal diameter $14 \%$ low enrichment uranium oxide/uranium carbide kernels (LEUCO). The TRISO-coatings consist of a 50\% dense carbon buffer layer (100 $\mu \mathrm{m}$ nominal thickness) followed by a dense inner pyrocarbon layer (40 $\mu \mathrm{m}$ nominal thickness) followed by a SiC layer (35 $\mu$ m nominal thickness) followed by another dense outer pyrocarbon layer ( $40 \mu \mathrm{m}$ nominal thickness). For this variant, argon was added to the hydrogen fluidization gas during $\mathrm{SiC}$ deposition.

The AGR-2 Fuel Specification (INL SPC-923) provides the requirements necessary for acceptance of the fuel manufactured for the AGR-2 irradiation test. The bulk of the kernels and coated particle acceptance testing was performed at $\mathrm{B} \& \mathrm{~W}$ and is not contained in this report. Sample NP-B7979 was sent to ORNL for supplemental characterization. The procedures for the limited characterization and qualification of the particles performed at ORNL are outlined in ORNL product inspection plan AGR-CHAR-PIP-09. The BAFo equivalent optical anisotropies of the inner and outer pyrocarbon layers are reported on Inspection Report Form IRF-09, with a determination as to whether the particle batch satisfied the specified parameters for this property. The batch was found to satisfy the AGR-2 Fuel Specification SPC-923, Rev. 1 for IPyC and OPyC anisotropy.

Also provided in this data package are data on the true BAFo, average particle weight, OPyC open porosity, and $\mathrm{SiC}$ soot inclusion defect fraction. True BAFo is calculated as $(1+\mathrm{N}) /(1-\mathrm{N})$, where $\mathrm{N}$ is the diattenuation. This differs from equivalent $\mathrm{BAFo}=1+3 \mathrm{~N}$, which is the calculation used by the fuel specification to allow comparison to historical measurements. Average OPyC open porosity was determined using a single sample to be $0.27 \mathrm{ml} / \mathrm{m}^{2}$. One $\mathrm{SiC}$ soot inclusion was found in a sample of 4746 particles. This corresponds to $<1 \mathrm{E}-3$ defect fraction at $95 \%$ confidence. 
Inspection Report Form IRF-09: AGR-2 Coated Particles

Procedure: AGR-CHAR-PIP-09 Rev. 0

Coated particle composite ID: G73J-14-93074A

Coated particle composite description: $A G R-2$ Variant particle batch

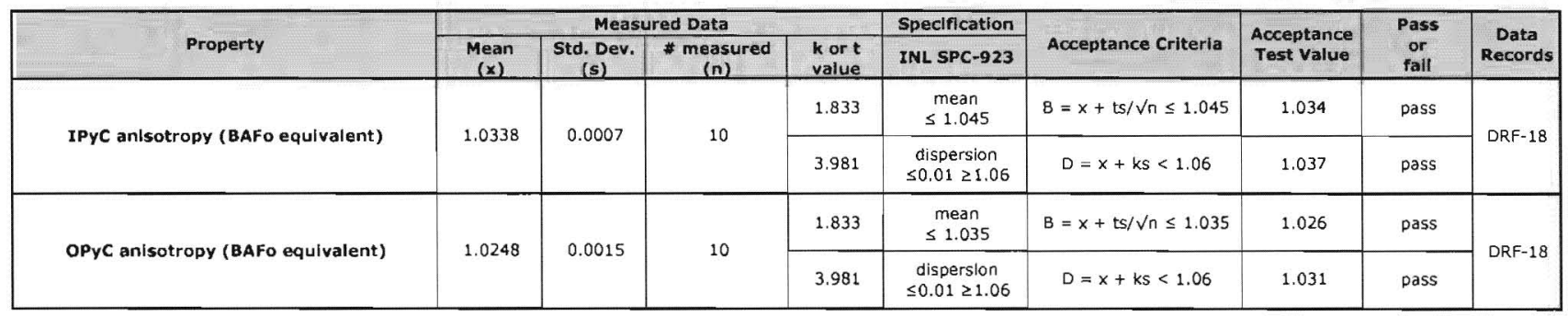
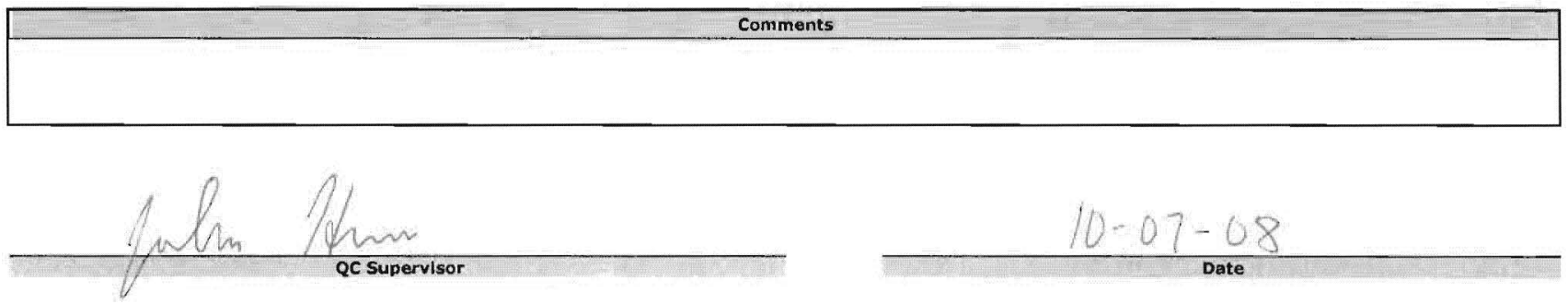

Accept coated particle composite (Yes or No):

Yes
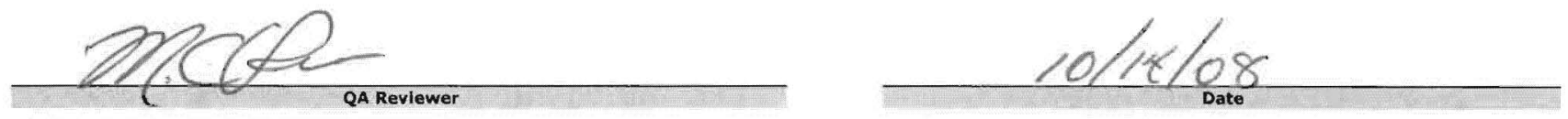
Data Report Form DRF-18A: Measurement of Pyrocarbon Anisotropy using the 2-MGEM - IPYC

\begin{tabular}{|r|l|}
\hline Procedure: & AGR-CHAR-DAM-18 Rev. 1 \\
\hline Operator: & G. E. Jellison \\
\hline Mount ID: & M08090201 \\
\hline Sample ID: & NP-B7979 \\
\hline Sample Description: & From G73J-14-93074A AGR-2 Variant batch \\
\hline Folder containing data: & \IMc-agr\AGR\2-MGEM\R080090501\} $\\
{\hline}$
\end{tabular}

\begin{tabular}{|c|c|c|c|c|c|c|c|}
\hline \multirow{2}{*}{ Particle \# } & \multirow{2}{*}{$\begin{array}{c}\text { Grid } \\
\end{array}$} & Position & Average & St. Dev. & Ave. Error & \multicolumn{3}{c|}{ Equivalent BAFo $=1+3 \mathrm{~N}$} \\
\cline { 3 - 8 } & 4,4 & 0.0115 & 0.0021 & 0.0006 & 1.0345 & 0.0063 & 0.0018 \\
\hline \hline 1 & 4,5 & 0.0114 & 0.0023 & 0.0006 & 1.0342 & 0.0069 & 0.0018 \\
\hline 2 & 4,6 & 0.0112 & 0.0021 & 0.0006 & 1.0336 & 0.0063 & 0.0018 \\
\hline 3 & 5,4 & 0.0114 & 0.0022 & 0.0006 & 1.0342 & 0.0066 & 0.0018 \\
\hline 4 & 5,5 & 0.0111 & 0.0023 & 0.0005 & 1.0333 & 0.0069 & 0.0015 \\
\hline 5 & 6,4 & 0.0113 & 0.0022 & 0.0005 & 1.0339 & 0.0066 & 0.0015 \\
\hline 6 & 6,5 & 0.0116 & 0.0019 & 0.0006 & 1.0348 & 0.0057 & 0.0018 \\
\hline 7 & 6,6 & 0.0109 & 0.0021 & 0.0006 & 1.0327 & 0.0063 & 0.0018 \\
\hline 8 & 4,7 & 0.0113 & 0.0021 & 0.0005 & 1.0339 & 0.0063 & 0.0015 \\
\hline 9 & 5,7 & 0.0110 & 0.0024 & 0.0006 & 1.0330 & 0.0072 & 0.0018 \\
\hline 10 & & 0.0113 & 0.0022 & 0.0006 & 1.0338 & 0.0065 & 0.0017 \\
\hline
\end{tabular}

Mean of average BAFo per particle: 1.0338 Standard deviation of average BAFo per particle: 0.0007

\section{Comments}
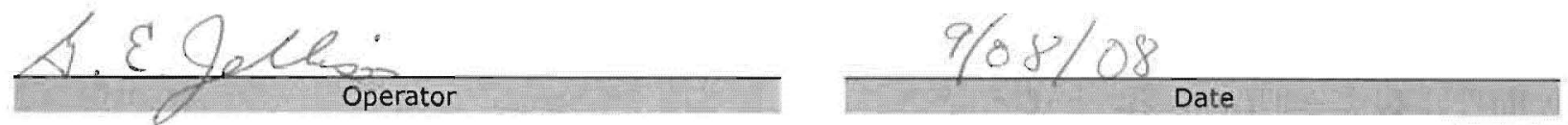


\section{Data Report Form DRF-18B: Measurement of Pyrocarbon Anisotropy using the 2-MGEM - OPyC}

\begin{tabular}{|r|l|}
\hline Procedure: & AGR-CHAR-DAM-18 Rev. 1 \\
\hline Operator: & G. E. Jellison \\
\hline Mount ID: & M08090201 \\
\hline Sample ID: & NP-B7979 \\
\hline Sample Description: & From G73J-14-93074A AGR-2 Variant batch \\
\hline Folder containing data: & IIMc-agr\AGR\2-MGEM $\backslash$ R080090501 \\
\hline
\end{tabular}

\begin{tabular}{|c|c|c|c|c|c|c|c|}
\hline \multirow{2}{*}{ Particle \# } & \multirow{2}{*}{$\begin{array}{c}\text { Grid } \\
\text { Position }\end{array}$} & \multicolumn{3}{|c|}{ Diattenuation } & \multicolumn{3}{|c|}{ Equivalent $\mathrm{BAFo}=1+3 \mathrm{~N}$} \\
\hline & & Average & St. Dev. & Ave. Error & Average & St. Dev. & Ave. Error \\
\hline 1 & 4,4 & 0.0076 & 0.0020 & 0.0006 & 1.0228 & 0.0060 & 0.0018 \\
\hline 2 & 4,5 & 0.0078 & 0.0020 & 0.0006 & 1.0234 & 0.0060 & 0.0018 \\
\hline 3 & 4,6 & 0.0084 & 0.0022 & 0.0005 & 1.0252 & 0.0066 & 0.0015 \\
\hline 4 & 5,4 & 0.0091 & 0.0023 & 0.0006 & 1.0273 & 0.0069 & 0.0018 \\
\hline 5 & 5,5 & 0.0083 & 0.0023 & 0.0006 & 1.0249 & 0.0069 & 0.0018 \\
\hline 6 & 6,4 & 0.0088 & 0.0021 & 0.0005 & 1.0264 & 0.0063 & 0.0015 \\
\hline 7 & 6,5 & 0.0079 & 0.0022 & 0.0006 & 1.0237 & 0.0066 & 0.0018 \\
\hline 8 & 6,6 & 0.0079 & 0.0024 & 0.0005 & 1.0237 & 0.0072 & 0.0015 \\
\hline 9 & 4,7 & 0.0081 & 0.0022 & 0.0005 & 1.0243 & 0.0066 & 0.0015 \\
\hline 10 & 5,7 & 0.0087 & 0.0021 & 0.0006 & 1.0261 & 0.0063 & 0.0018 \\
\hline \multicolumn{2}{|c|}{ Average } & 0.0083 & 0.0022 & 0.0006 & 1.0248 & 0.0065 & 0.0017 \\
\hline
\end{tabular}

Mean of average BAFo per particle: 1.0248 Standard deviation of average BAFo per particle: 0.0015

\section{Comments}

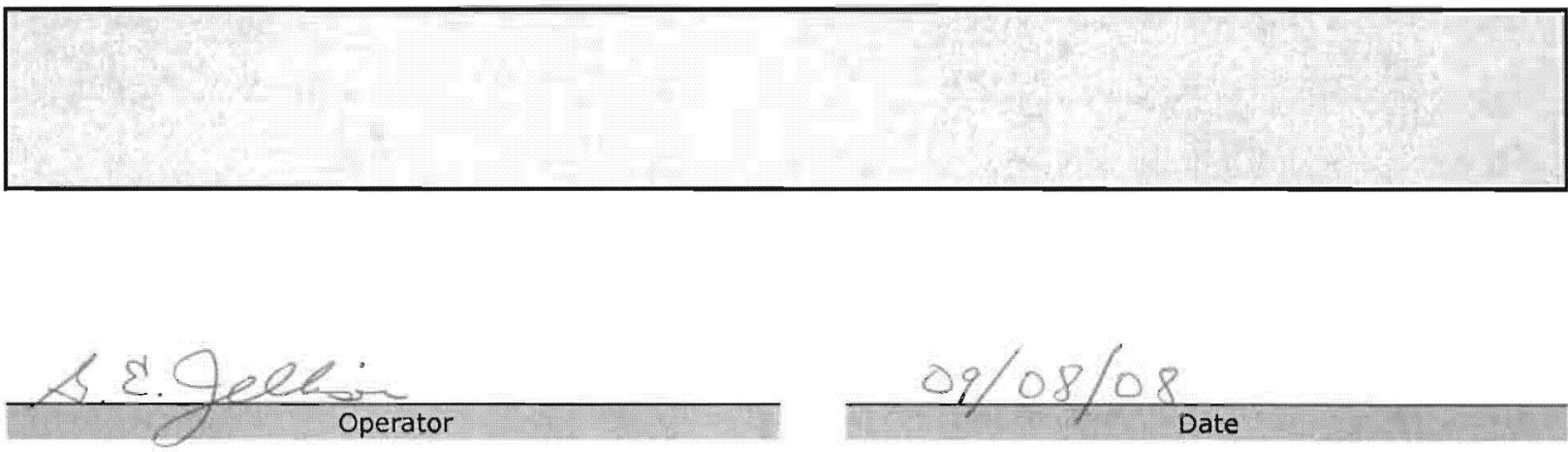


Data Report Form DRF-18A: Measurement of Pyrocarbon Anisotropy using the 2-MGEM - IPyC

\begin{tabular}{|r|l|}
\hline Procedure: & AGR-CHAR-DAM-18 Rev. 1 \\
\hline Operator: & G. E. Jellison \\
\hline Mount ID: & M08090201 \\
\hline Sample ID: & NP-B7979 \\
\hline Sample Description: & From G73J-14-93074A AGR-2 Variant batch \\
\hline Folder containing data: & \Imc-agr\AGR\2-MGEM\R080090501\} $\\
{\hline}$
\end{tabular}

\begin{tabular}{|c|c|c|c|c|c|c|c|}
\hline \multirow{2}{*}{ Particle \# } & \multirow{2}{*}{$\begin{array}{c}\text { Grid } \\
\text { Position }\end{array}$} & \multicolumn{3}{|c|}{ Diattenuation } & \multicolumn{3}{|c|}{ True $B A F O=(1+N) /(1-N)$} \\
\hline & & Average & St. Dev. & Ave. Error & Average & St. Dev. & Ave. Error \\
\hline 1 & 4,4 & 0.0115 & 0.0021 & 0.0006 & 1.0233 & 0.0043 & 0.0012 \\
\hline 2 & 4,5 & 0.0114 & 0.0023 & 0.0006 & 1.0231 & 0.0047 & 0.0012 \\
\hline 3 & 4,6 & 0.0112 & 0.0021 & 0.0006 & 1.0227 & 0.0043 & 0.0012 \\
\hline 4 & 5,4 & 0.0114 & 0.0022 & 0.0006 & 1.0231 & 0.0045 & 0.0012 \\
\hline 5 & 5,5 & 0.0111 & 0.0023 & 0.0005 & 1.0224 & 0.0047 & 0.0010 \\
\hline 6 & 6,4 & 0.0113 & 0.0022 & 0.0005 & 1.0229 & 0.0045 & 0.0010 \\
\hline 7 & 6,5 & 0.0116 & 0.0019 & 0.0006 & 1.0235 & 0.0039 & 0.0012 \\
\hline 8 & 6,6 & 0.0109 & 0.0021 & 0.0006 & 1.0220 & 0.0043 & 0.0012 \\
\hline 9 & 4,7 & 0.0113 & 0.0021 & 0.0005 & 1.0229 & 0.0043 & 0.0010 \\
\hline 10 & 5,7 & 0.0110 & 0.0024 & 0.0006 & 1.0222 & 0.0049 & 0.0012 \\
\hline \multicolumn{2}{|c|}{ Average } & 0.0113 & 0.0022 & 0.0006 & 1.0228 & 0.0044 & 0.0012 \\
\hline
\end{tabular}

Mean of average BAFo per particle: 1.0228

Standard deviation of average BAFo per particle: 0.0005

\section{Comments}


Data Report Form DRF-18B: Measurement of Pyrocarbon Anisotropy using the 2-MGEM - OPYC

\begin{tabular}{|r|l|}
\hline Procedure: & AGR-CHAR-DAM-18 Rev. 1 \\
\hline Operator: & G. E. Jellison \\
\hline Mount ID: & M08090201 \\
\hline Sample ID: & NP-B7979 \\
\hline Sample Description: & From G73J-14-93074A AGR-2 Variant batch \\
\hline Folder containing data: & IIMc-agr\AGR $\backslash 2-M G E M \backslash R 080090501 \backslash$ \\
\hline
\end{tabular}

\begin{tabular}{|c|c|c|c|c|c|c|c|}
\hline \multirow{2}{*}{ Particle \# } & \multirow{2}{*}{$\begin{array}{c}\text { Grid } \\
\text { Position }\end{array}$} & \multicolumn{3}{|c|}{ Diattenuation } & \multicolumn{3}{|c|}{ True BAFo $=(1+N) /(1-N)$} \\
\hline & & Average & St. Dev. & Ave. Error & Average & St. Dev. & Ave. Error \\
\hline 1 & 4,4 & 0.0076 & 0.0020 & 0.0006 & 1.0153 & 0.0041 & 0.0012 \\
\hline 2 & 4,5 & 0.0078 & 0.0020 & 0.0006 & 1.0157 & 0.0041 & 0.0012 \\
\hline 3 & 4,6 & 0.0084 & 0.0022 & 0.0005 & 1.0169 & 0.0045 & 0.0010 \\
\hline 4 & 5,4 & 0.0091 & 0.0023 & 0.0006 & 1.0184 & 0.0047 & 0.0012 \\
\hline 5 & 5,5 & 0.0083 & 0.0023 & 0.0006 & 1.0167 & 0.0047 & 0.0012 \\
\hline 6 & 6,4 & 0.0088 & 0.0021 & 0.0005 & 1.0178 & 0.0043 & 0.0010 \\
\hline 7 & 6,5 & 0.0079 & 0.0022 & 0.0006 & 1.0159 & 0.0045 & 0.0012 \\
\hline 8 & 6,6 & 0.0079 & 0.0024 & 0.0005 & 1.0159 & 0.0049 & 0.0010 \\
\hline 9 & 4,7 & 0.0081 & 0.0022 & 0.0005 & 1.0163 & 0.0045 & 0.0010 \\
\hline 10 & 5,7 & 0.0087 & 0.0021 & 0.0006 & 1.0176 & 0.0043 & 0.0012 \\
\hline \multicolumn{2}{|c|}{ Average } & 0.0083 & 0.0022 & 0.0006 & 1.0167 & 0.0044 & 0.0011 \\
\hline
\end{tabular}

Mean of average BAFo per particle: 1.0167 Standard deviation of average BAFo per particle: 0.0010

\section{Comments}
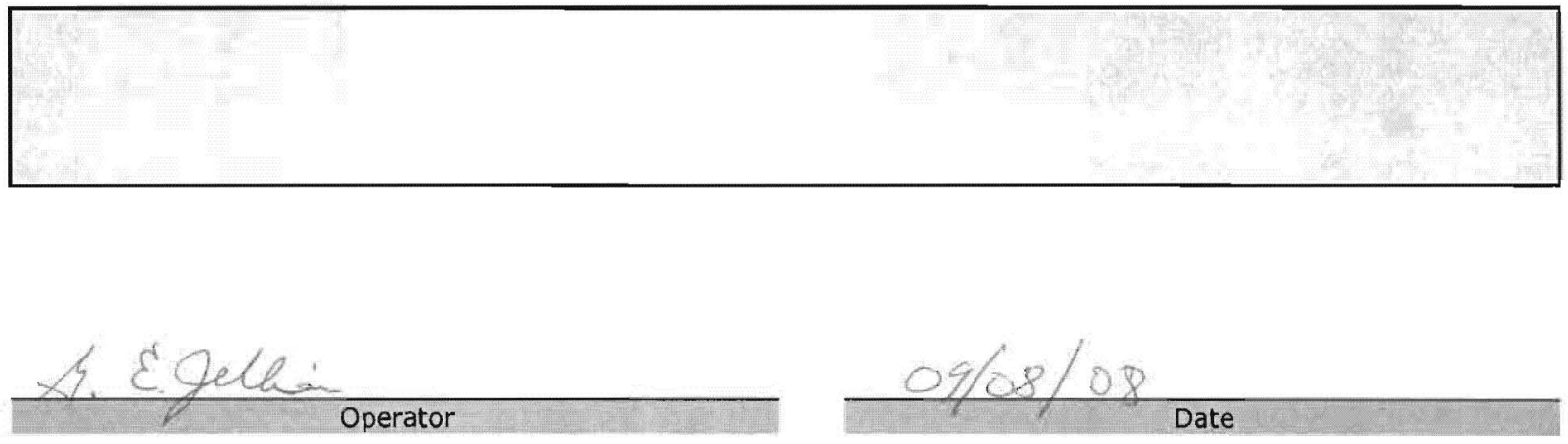
Data Report Form DRF-22: Estimation of Average Particle Welght

\begin{tabular}{|c|c|}
\hline Procedure: & AGR-CHAR-DAM-22 Rev. 1 \\
\hline Operator: & Dixle Barker \\
\hline Particle Lot ID: & NP-B7979 \\
\hline Particle Lot Description: & From G73J-14-93074A AGR-2 Variant Batch \\
\hline Filename: & IImc-agr\AGR\ParticleWeight|W08082802_DRF22R1.xIs \\
\hline
\end{tabular}

\begin{tabular}{|r|c|c|c|c|c|}
\hline & Sample 1 & Sample 2 & Sample 3 & Sample 4 & Sample 5 \\
\hline Weight of particles (g): & 0.1413 & 0.1454 & 0.1358 & 0.1447 & 0.1647 \\
\hline Number of particles: & 136 & 139 & 131 & 139 & 159 \\
\hline Average weight/particle (g): & $1.039 \mathrm{E}-03$ & $1.046 \mathrm{E}-03$ & $1.037 \mathrm{E}-03$ & $1.041 \mathrm{E}-03$ & $1.036 \mathrm{E}-03$ \\
\hline
\end{tabular}

Mean average weight/particle $(\mathrm{g}): 1.040 \mathrm{E}-03$

Standard error In mean average weight/particle $(\mathrm{g}): 1.83 \mathrm{E}-06$
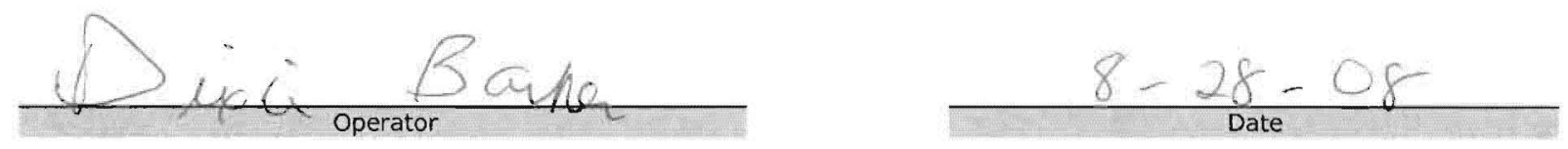
Data Report Form DRF-31: Measurement of Open Porosity using a Mercury Porosimeter

\begin{tabular}{|c|c|}
\hline Procedure: & AGR-CHAR-DAM-31 Rev. 1 \\
\hline Operator: & S. D. Nunn \\
\hline Coated particle batch ID: & NP-B7979-D01 \\
\hline Batch Description: & From G73J-14-93074A AGR-2 Variant batch \\
\hline Thermocouple Explration Date: & $5 / 15 / 09$ \\
\hline Penetrometer Expiration Date: & $7 / 10 / 08$ \\
\hline Completed DRF Fllename: & 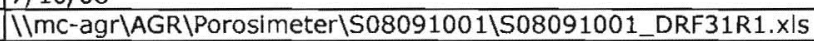 \\
\hline
\end{tabular}

Mean average weight/particle $(g): 1.04 \mathrm{E}-03$

\begin{tabular}{ll|l} 
Standard error In mean average weight/particle $(\mathrm{g}):$ & $1.83 \mathrm{E}-06$
\end{tabular}

\begin{tabular}{|r|l|}
\hline Weight of particles $(\mathrm{g}):$ & 3.9551 \\
\hline Approximate number of particles: & 3803 \\
\hline Uncertalnty in number of particles: & 7 \\
\hline Total envelope volume of sample $(\mathrm{cc}):$ & 1.318 \\
\hline Average envelope volume/particle $(\mathrm{cc}):$ & $3.47 \mathrm{E}-04$ \\
\hline Sample envelope density $(\mathrm{g} / \mathrm{cc}):$ & 3.001 \\
\hline
\end{tabular}

Average particle diameter (microns): $8.71 \mathrm{E}+02$

Average surface area/particle $(\mathrm{cm} 2): 2.39 \mathrm{E}-02$

Total sample surface area $(\mathrm{cm} 2): 9.07 \mathrm{E}+01$

Intruded mercury volume from $250-10,000$ psia (cc): $2.40 \mathrm{E}-03$

Open porosity $(\mathrm{ml} / \mathrm{m} 2): 2.65 E-01$ 
Data Report Form DRF-32: Counting of Particles with SiC Soot Inclusion Defects by Visual Inspection

\begin{tabular}{|c|c|}
\hline Procedure: & AGR-CHAR-DAM-32 Rev. 0 \\
\hline Operator: & Fred Montgomery \\
\hline Sample ID: & NP-B7979-E01 \\
\hline Sample Description: & From G73J-14-93074A AGR-2 Variant Batch \\
\hline Folder containing images: & 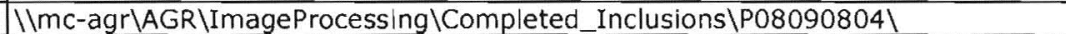 \\
\hline DRF filename: & 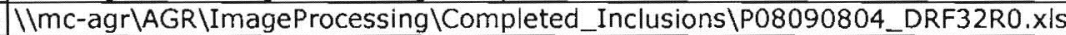 \\
\hline
\end{tabular}

Mean average weight/particle $(\mathrm{g}): 1.04 \mathrm{E}-03$

Uncertainty in average weight/particle $(\mathrm{g}): 1.83 \mathrm{E}-06$

Weight of sample of particles $(\mathrm{g}): 4.936$

Approximate number of particles in sample: 4746

Uncertainty in number of particles in sample: 8

Number of particles with SiC soot inclusion defects: 1

\section{Comments}

$1 / 4746$ corresponds to $<1 \mathrm{E}-3$ defect fraction at $95 \%$ confidence.

The one defect identified was marginal. It was just a few spots chipped out at about the same radius. It was, however, associated with the presence of several darkfield bands, and probably indicates a region of weaker SiC.

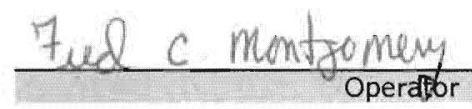

$10-14-08$

Date 
From frame 66, mount 4 of NP-B7979-E01, G73J-14-93074A AGR-2 Variant batch.

This is the only particle out of 4746 identifed with a bright field anomaly in the SiC. The defect is not severe. It was, however, associated with the presence of several darkfield bands, and probably indicates a region of weaker $\mathrm{SiC}$.

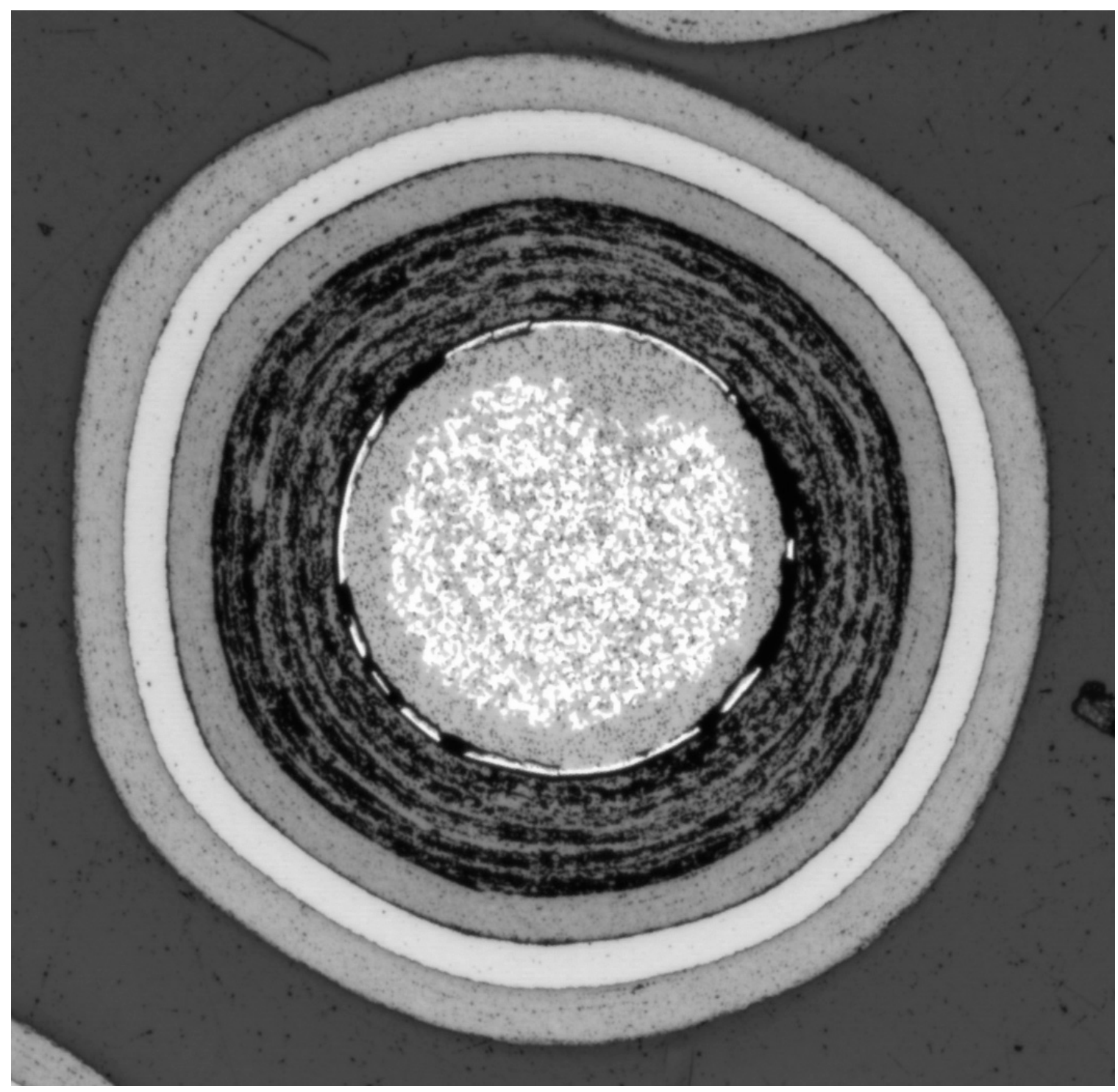

